\title{
Light-Trail Assignment in WDM Optical Networks
}

\author{
Bin Wu and Kwan L. Yeung \\ Dept. of Electrical and Electronic Engineering \\ The University of Hong Kong \\ Pokfulam, Hong Kong \\ E-mail: \{binwu, kyeung\}@eee.hku.hk
}

\begin{abstract}
Light-trail has emerged as a promising candidate for enabling IP over WDM networks. The problem of static lighttrail assignment is to find a set of light-trails to cover the given traffic demands, such that the total number of light-trails required is minimized. Because of the power loss caused by splitting at each hop, the length of a light-trail is limited. Existing light-trail assignment algorithms adopt ILP (Integer Linear Programming) approach. Due to the high complexity of ILP, such algorithms are not scalable. In this paper, we propose an efficient heuristic algorithm LTA (Light-Trail Assignment) to solve this problem. In LTA, each light-trail is judiciously assigned based on the request discreteness, the shortest path length and the traffic volume of each request. A reference node mechanism is also designed to enhance the solution. Numerical results show that LTA always returns sub-optimal solutions.
\end{abstract}

Keywords-Heuristic; integer linear programming (ILP); lighttrail assignment; wavelength division multiplexing (WDM).

\section{INTRODUCTION}

IP over WDM is an appealing architecture for meeting the ever-increasing data demands in the Internet. The bandwidth of a WDM wavelength is usually 10Gbps, whereas the bandwidth required by sub-rate traffic varies from STS-1 (51.84 Mbps) to the full wavelength capacity. In traditional optical flow/circuit switching (OFS) networks, an all-optical lightpath is set up between two communicating nodes using a dedicated wavelength. When the traffic load is light, the wavelength capacity is under-utilized. To improve the wavelength utilization, optical packet switching (OPS) [1] and optical burst switching (OBS) [2] are proposed. However, such techniques require either expensive or immature optical components such as optical buffers and fast optical switching.

Recently, light-trail [3] has been proposed as a promising technique for IP over WDM. A light-trail is a unidirectional optical bus between a convener node (i.e. light-trail head) and an end node. It is implemented using a wavelength, and allows the intermediate nodes to share the bandwidth by adding or dropping traffic, provided that the total traffic load carried is not more than the bandwidth of the wavelength. An upstream node can send data to any downstream node, and a downstream node only accepts those packets destined to it. At each intermediate node, this is achieved by splitting a percentage of optical power and detecting the optical signals using a transponder. Meanwhile, the rest of the optical signals continue to propagate along the light-trail. A signaling mechanism is used to ensure conflict-free communications among the nodes [3]. Such a light-trail architecture is based on mature optical technologies. It improves wavelength utilization without using optical buffers, fast optical switching and O-E-O conversions at the intermediate nodes. Due to the power loss caused by splitting at each hop, the length of a light-trail is limited and is expected to be not more than 5 hops [4].

Light-trail attracts a lot of research interests [3-11]. The node architecture and signaling mechanisms are detailed in [34]. Performance evaluations can be found in [3-5]. ILP formulations are given in [6-7] for light-trail assignment. Traffic grooming [9], dynamic routing and protection [10] are also studied in light-trail networks. Besides, bidirectional lighttrail (BDLT) is recently proposed to enhance the fairness performance [11].

In this paper, we propose an efficient heuristic LTA (LightTrail Assignment) to assign light-trails under a given traffic matrix. To the best of our knowledge, LTA is the first heuristic designed so far. Compared to the ILP approach, LTA is more scalable and is shown to always return a sub-optimal solution. The rest of the paper is organized as follows. In Section II, the light-trail assignment problem is formulated. LTA is proposed in Section III. Section IV shows the numerical results and Section V gives some discussion. We conclude the paper in Section VI.

\section{Light-Trail ASSIGNMENT Problem}

For a network with $N$ nodes, an $N \times N$ traffic matrix $\boldsymbol{T}=\left\{t_{i j}\right\}$ is used to denote the traffic demands among all node pairs. Let $C$ be the capacity of a wavelength and assume each individual demand/request $t_{i j} \leq C$. The light-trail assignment problem is to determine a minimum set of light-trails to carry all the requests in $\boldsymbol{T}$. For each light-trail, its length (measured in hops) must not exceed a predefined value $L_{\max }$, and the total traffic carried must not exceed $C$.

Let $h_{i j}$ be the length of the shortest path between nodes $i$ and $j$. If $h_{i j} \leq L_{\max }$, flow $t_{i j}$ is carried by a single light-trail. If $h_{i j}>L_{\max }$, more than one concatenated light-trails are required. In the latter case, each light-trail end/head node introduces an $\mathrm{O}-\mathrm{E}-\mathrm{O}$ conversion. So it is necessary to minimize the number of concatenated light-trails required. While this can be achieved in many ways, we reuse the traffic matrix preprocessing in [6], which is reproduced in Fig. 1. The idea is to greedily break the shortest path from node $i$ to node $j$ into several segments with length $L_{\max }$ each, and the length of the

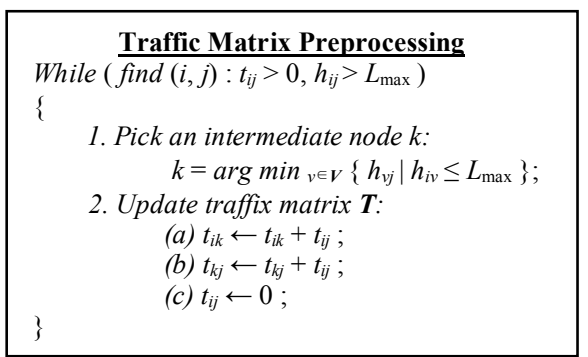

Fig. 1. Traffic matrix preprocessing ( $\boldsymbol{V}$ is the set of all the nodes).

This work is supported by Hong Kong Research Grant Council Earmarked Grant HKU 7150/04E. 
last segment can be smaller than $L_{\max }$. Accordingly, the value $t_{i j}$ is added to the existing traffic volume between the two end nodes of each segment.

Two key issues are involved in the light-trail assignment problem: determining the set of requests to be carried by each light-trail, and minimizing the total number of light-trails required. Light-trail assignment is similar to the knapsack and bin packing problems, which are both NP-hard. It differs from them in having the extra light-trail length constraint $\left(L_{\max }\right)$. This makes it even harder to solve [6].

Due to the intractability of the problem, existing algorithms [6-7] rely on ILP. In [6], all the paths with hop-count $L_{\max }$ are first found by breath first search. They form a set of candidate trails $\boldsymbol{L}_{\boldsymbol{T}}$. Then, a subset of $\boldsymbol{L}_{\boldsymbol{T}}$ is returned by ILP as the lighttrails. However, the ILP approach is not scalable.

\section{LTA ALGORITHM}

\section{A. Observations}

To minimize the required number of light-trails, intuitively we can design a greedy algorithm to let a light-trail carry as much traffic as it can. But this is not enough as illustrated in Fig. 2. Assume $L_{\max }=3$ and $C=48$. The number next to each dashed arrow in Fig. 2 gives the value of $t_{i j}$. In Fig. 2a, if we first use two light-trails $0 \rightarrow 1 \rightarrow 2 \rightarrow 3$ and $4 \rightarrow 5 \rightarrow 6 \rightarrow 7$ to carry the requests $\left\{t_{01}, t_{12}, t_{23}\right\}$ and $\left\{t_{45}, t_{56}, t_{67}\right\}$ in a greedy manner, then the remaining requests $t_{34}$ and $t_{70}$ need two additional light-trails. This is because the length of a light-trail must be no more than $L_{\max }=3$ hops, and $t_{34}, t_{70}$ are not close enough to share a single light-trail. However, if light-trails $0 \rightarrow 1 \rightarrow 2 \rightarrow 3$ and $3 \rightarrow 4 \rightarrow 5 \rightarrow 6$ are used to carry $\left\{t_{01}, t_{12}, t_{23}\right\}$ and $\left\{t_{34}, t_{45}, t_{56}\right\}$, then the remaining requests $t_{67}$ and $t_{70}$ can share a common light-trail. This gives a better solution of three light-trails only. This example shows the importance of request discreteness.

In Fig. $2 \mathrm{~b}$, if we first pack $\left\{t_{01}, t_{12}, t_{23}\right\}$ onto light-trail $0 \rightarrow 1 \rightarrow 2 \rightarrow 3$, then the remaining requests $t_{03}$ and $t_{42}$ require two additional light-trails. Note that $h_{03}$ and $h_{42}$ are larger than $h_{i j}$ of the other three requests. If we first pack the longest request $t_{03}$ with $t_{12}$ and $t_{23}$ onto light-trail $0 \rightarrow 1 \rightarrow 2 \rightarrow 3$, then the request $t_{01}$ can take a detour to share light-trail $0 \rightarrow 4 \rightarrow 1 \rightarrow 2$ with $t_{42}$. The latter case requires one less light-trail than the former. This example shows that it is better to consider requests $t_{i j}$ with large $h_{i j}$ first, because a request with smaller $h_{i j}$ usually has more flexibility in sharing a common light-trail with others.

In Fig. 2c, we first consider $t_{02}$ because $h_{02}$ is the largest. However, if $t_{02}$ and $t_{23}$ are packed onto light-trail $0 \rightarrow 1 \rightarrow 2 \rightarrow 3$, then the remaining bandwidth of this light-trail $(48-28=20)$ is not sufficient to carry $t_{01}(=25)$. As a result, $t_{40}$ and $t_{01}$ need two additional light-trails. However, if $t_{02}$ is packed with $t_{40}$ or $t_{01}$, the remaining requests can share a common light-trail. This example shows that we should first accommodate those requests with large $t_{i j}$, as it is generally easier for a request with smaller $t_{i j}$ to share a common light-trail with others.

We argue that the importance of the above three factors are ranked in the same order as they were discussed. If some requests are far from each other, it is impossible for them to share a common light-trail regardless of the values of $h_{i j}$ and $t_{i j}$. So, request discreteness has the largest impact. On the other hand, because of the hop constraint $L_{\max }, h_{i j}$ has a larger impact than $t_{i j}$. For example, in Fig. $2 \mathrm{~b}$, no matter what are the values

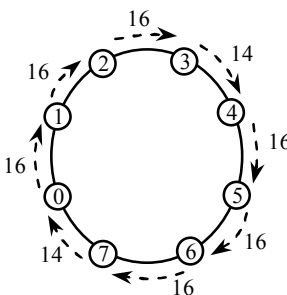

(a)

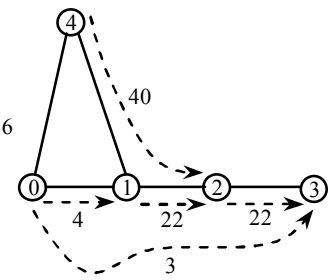

(b)

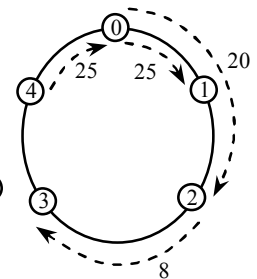

(c)
Fig. 2. Three examples with $L_{\max }=3$ and $C=48$.

of $t_{03}$ and $t_{42}$, it is impossible for them to share a light-trail of $L_{\max }=3$. We can consider the flow volume $t_{i j}$ only when it is possible to pack the requests onto a common light-trail.

\section{B. General Idea of LTA}

We first introduce the notion of reference node. A reference node $r$ is a node based on which we can calculate how far a request $t_{i j}$ is away from it. The distance between $t_{i j}$ and $r$ is defined as

$$
d_{i j}^{r}=h_{i r}+h_{j r} .
$$

In LTA, we first consider the request with the largest $d_{i j}{ }^{r}$. If a request $t_{i j}$ is carried by a light-trail, we set its $d_{i j}^{r}$ to 0 . As the number of determined light-trails increases, the maximum value of $d_{i j}^{r}$ decreases, and the not-yet-carried requests gradually converge to the reference node. This helps to address the request discreteness issue. If multiple requests have the same $d_{i j}{ }^{r}$, we consider the one with the largest $h_{i j}$ first. Similarly, if multiple requests have the same $d_{i j}{ }^{r}$ and $h_{i j}$, the one with the largest traffic volume $t_{i j}$ is considered first.

Assume $\boldsymbol{L}_{\boldsymbol{T}}$ is the candidate trail set found by breadth first search, and $t_{a b}$ is the farthest request from $r$ (or the most crucial request). We find a subset of trails $\boldsymbol{L}_{S} \subseteq \boldsymbol{L}_{\boldsymbol{T}}$ that can carry $t_{a b}$. For each trail $l \in \boldsymbol{L}_{\boldsymbol{S}}$, we invoke a packing process pack(l) (to be discussed later). It returns a tentative packing scheme $\boldsymbol{P}(\boldsymbol{l})$ which contains all the requests (denoted by node pairs) that can be carried by $l$. Then, the best one $l_{\text {best }}$ is determined from (2) as a light-trail, and the packing scheme for $l_{\text {best }}$ is stored in $\boldsymbol{P}\left(\boldsymbol{l}_{\text {best }}\right)$.

$$
l_{\text {best }}=\left.\arg \max _{l \in L_{S}}\{\text { sum_t }\}\right|_{\max _{\in \in L_{S}}\{\text { sum_h } h\}}
$$

where

$$
\begin{aligned}
& \text { sum_ }_{-} h=\sum_{(i, j) \in \boldsymbol{P}(l)} h_{i j}, \\
& \text { sum_t }=\sum_{(i, j) \in \boldsymbol{P}(\boldsymbol{l})} t_{i j} .
\end{aligned}
$$

From $(2) \sim(4)$, the trail with the maximum sum $h$ is chosen as $l_{\text {best }}$. In case of multiple choices, the one with the heaviest traffic load (or the maximum sum_t) is chosen. Then, both $t_{i j}$ and $h_{i j}$ are set to 0 for $(i, j) \in \boldsymbol{P}\left(\boldsymbol{l}_{\text {best }}\right)$. We repeat this process until all $t_{i j} \in \boldsymbol{T}$ are carried.

However, it is difficult to determine the best reference node $r$. A simple technique is to try out every node as $r$ and get a solution $\boldsymbol{S o l}_{\boldsymbol{r}}$. Then the best solution is chosen among $\left\{\boldsymbol{S o l}_{\boldsymbol{r}}\right.$ $0 \leq r<N\}$. This simplifies the problem, and provides a way for $N$ solutions. The cost is an extra factor of $N$ in time complexity.

\section{Packing Process}

We apply the packing process $\operatorname{pack}(l)$ to each $l \in \boldsymbol{L}_{\boldsymbol{S}}$. The total possible number of requests that can be packed onto $l$ is 


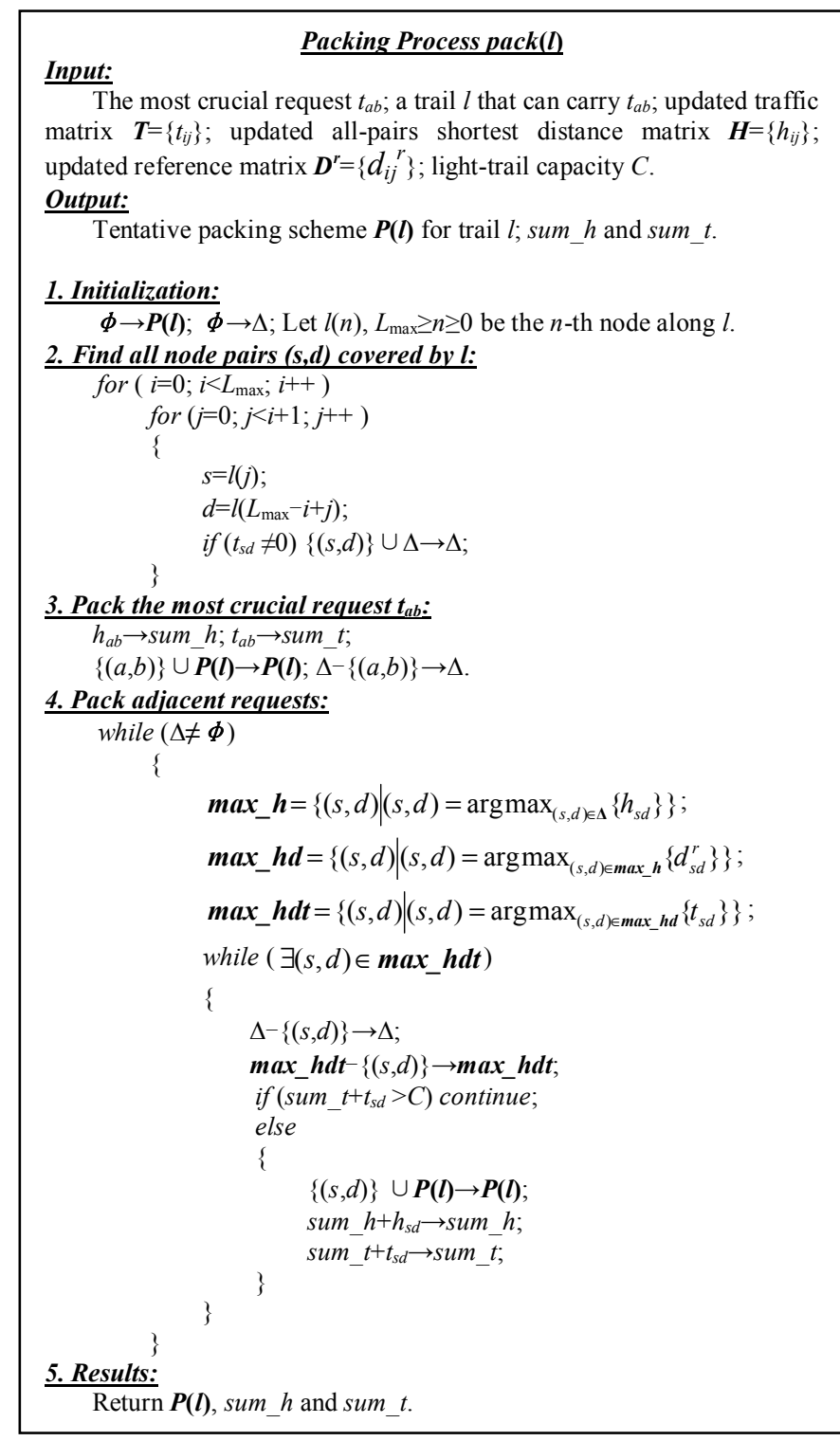

Fig. 3. Packing process pack(l). The inputs $\boldsymbol{T}, \boldsymbol{H}$ and $\boldsymbol{D}^{r}$ are updated in Fig. 4.

$$
\left(\begin{array}{c}
L_{\max }+1 \\
2
\end{array}\right)=\sum_{k=1}^{L_{\max }} k=\frac{1}{2}\left(L_{\max }^{2}+L_{\max }\right),
$$

In $\operatorname{pack}(l), t_{a b}$ is first packed onto $l$. Then adjacent requests are considered in the descending order of $h_{i j}$. In case of multiple choices, $d_{i j}{ }^{r}$ and $t_{i j}$ are compared in turn, and the requests with the largest $d_{i j}{ }^{r}$ or $t_{i j}$ are packed first. Inside pack $(l)$, we give $h_{i j}$ higher priority than $d_{i j}{ }^{r}$ because $d_{i j}{ }^{r}$ has already been used to identify $t_{a b}$ before entering pack $(l)$.

The pseudo-code for pack $(l)$ is given in Fig. 3. It returns a tentative packing scheme $\boldsymbol{P}(\boldsymbol{l})$, and the values of sum $h$ and sum_t as defined in (3) \& (4).

\section{LTA Algorithm}

In LTA, we first use Floyd-Warshall algorithm [12] to calculate the all-pairs shortest distance matrix $\boldsymbol{H}=\left\{h_{i j}\right\}$. The traffic matrix preprocessing in Fig. 1 is used to ensure that all $t_{i j}$ satisfies $h_{i j} \leq L_{\max }$. Then, $N$ solutions $\left\{\boldsymbol{S o l}_{\boldsymbol{r}} \mid 0 \leq r<N\right\}$ are constructed based on each $r$, where the most crucial request is

\section{LTA ALGORITHM}

Input:

a) Light-trail length limit $L_{\max }$ and wavelength capacity $C$;

b) A network with nodes numbered from 0 to $N^{-1}$;

c) A traffic matrix $\boldsymbol{T}=\left\{t_{i j} \mid t_{i j} \leq C\right\}$.

Output:

A solution Sol containing a minimum set of light-trails, with a packing scheme for each light-trail.

\section{Step 1) Initialization:}

Use Floyd-Warshall algorithm to calculate the all-pairs shortest distance matrix $\boldsymbol{H}=\left\{h_{i j}\right\}$. Apply the traffic matrix preprocessing in Fig. 1 to $\boldsymbol{T}$. Find all candidate trails with hop-count $L_{\max }$ by breath first search and record them in the candidate trail set $\boldsymbol{L}_{T}$. Initialize $\boldsymbol{S o l}_{0}, \ldots, \boldsymbol{S o \boldsymbol { l } _ { N - 1 }}$ to null. Set $0 \rightarrow r, \boldsymbol{T} \rightarrow \boldsymbol{T}_{\text {bak }}$ and $\boldsymbol{H} \rightarrow \boldsymbol{H}_{b a k}$.

Step 2) Construct Sol :

a) Based on the reference node $r$ and formula (1), calculate the distance matrix $\boldsymbol{D}^{r}=\left\{d_{i j}{ }^{r}\right\}$ from $\boldsymbol{H}$. If $t_{i j}=0$ then set $0 \rightarrow d_{i j}{ }^{r}$.

b) Find the most crucial request $t_{a b}$ with the largest $d_{i j}{ }^{r}$. In case of multiple choices, consider the request with the largest $h_{i j}$ first. If multiple requests have the same values of $d_{i j}{ }^{r}$ and $h_{i j}$, choose the one with the largest $t_{i j}$.

c) Find a subset of trails $\boldsymbol{L}_{S} \subseteq \boldsymbol{L}_{T}$ that can carry $t_{a b}$.

d) Apply the packing process pack $(l)$ in Fig. 3 to each trail $l \in \boldsymbol{L}_{\boldsymbol{S}}$. Determine the best trail $l_{\text {best }}$ as a light-trail according to (2). Record $l_{\text {best }}$ and the corresponding packing scheme $\boldsymbol{P}\left(\boldsymbol{l}_{\text {best }}\right)$ to $\boldsymbol{S o l} \boldsymbol{r}$.

e) For each node pair $(i, j) \in \boldsymbol{P}\left(\boldsymbol{l}_{\text {best }}\right)$, set $0 \rightarrow t_{i j}, 0 \rightarrow h_{i j}$ and $0 \rightarrow d_{i j}{ }^{r}$.

f) If $\exists\left(t_{i j} \in \boldsymbol{T}, t_{i j} \neq 0\right)$, go to Step $\left.2 b\right)$. Otherwise continue.

g) Set $\boldsymbol{T}_{\text {bak }} \rightarrow \boldsymbol{T}, \boldsymbol{H}_{\text {bak }} \rightarrow \boldsymbol{H}$ and $r+1 \rightarrow r$. If $r<N$, go to Step 2a). Otherwise go to Step 3).

Step 3) Determine the final solution Sol:

Among the $N$ solutions $\left\{\boldsymbol{S o l}_{\boldsymbol{r}} \mid 0 \leq r<N\right\}$ obtained, choose the one with the minimum number of light-trails as the final solution $\boldsymbol{S o l}$.

Fig. 4. LTA algorithm.

considered first. Light-trails are determined from the packing process and formula (2). The final solution $\boldsymbol{S o l}$ is chosen from $\left\{\boldsymbol{S o l}_{r} \mid 0 \leq r<N\right\}$ to minimize the number of light-trails required. LTA algorithm is formally summarized in Fig. 4.

Assume that $\boldsymbol{L}_{\boldsymbol{T}}$ is already obtained. We consider the time complexity of Step 2) in LTA. In constructing a light-trail (Step $2 b) \sim 2 e$ )), at most $\left|\boldsymbol{L}_{T}\right|$ candidate trails are examined using the packing process, which considers $O\left(L_{\max }{ }^{2}\right)$ requests (see Fig. 3 and formula (5)) and $O\left(L_{\max }{ }^{2}\right)$ comparisons to determine a request for packing. Therefore, its complexity is $O\left(\left|\boldsymbol{L}_{T}\right| L_{\max }{ }^{4}\right)$. Since each light-trail carries at least one request and there are at most $N^{2}$ requests, the complexity for an $\boldsymbol{S o l}_{r}$ construction is $O\left(\left|\boldsymbol{L}_{T}\right| L_{\max }{ }^{4} N^{2}\right)$. The final solution $\boldsymbol{S o l}$ is chosen among $\left\{\boldsymbol{S o l}_{r}\right.$ $0 \leq r<N\}$. This gives Step 2) the complexity of $O\left(\left|\boldsymbol{L}_{T}\right| L_{\max }{ }^{4} N^{3}\right)$.

\section{NumericAl Results}

To compare the performance of LTA with ILP, we consider the examples shown in Fig. 5, where topology (a) and traffic matrix (g) are taken from [6], and traffic matrix (h) is obtained by enlarging the matrix in $(\mathrm{g})$. Particularly, traffic matrix $(\mathrm{g})$ is for topologies (a) $\sim(e)$ and $(\mathrm{h})$ is for topology (f). We assume $L_{\max }=4$ and $C=48$ for all examples.

TABLE I summarizes the results. The $2^{\text {nd }}$ and $3^{\text {rd }}$ rows give the number of light-trails required by ILP and LTA. The $4^{\text {th }}$ row shows the best $r$ for LTA. Performance gap between LTA and ILP is given in the $5^{\text {th }}$ row. The last two rows include the number of wavelengths/light-trails required at the mostheavily-loaded link. From TABLE I, we can see that LTA 


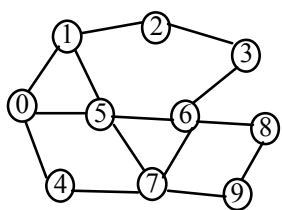

(a)

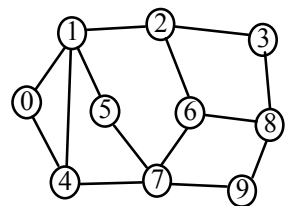

(b)

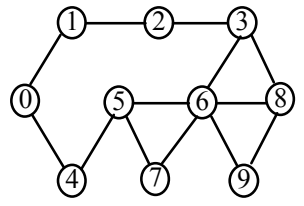

(c)

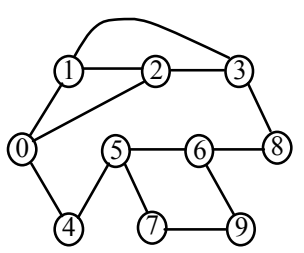

(d)

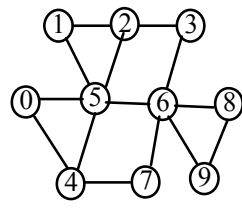

(e)

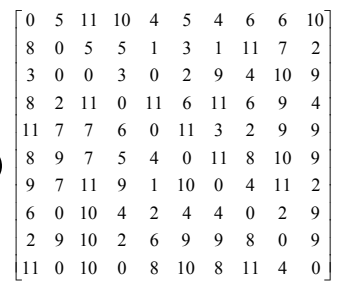

(g)

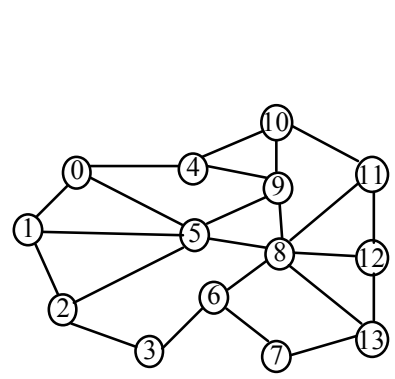

(f)

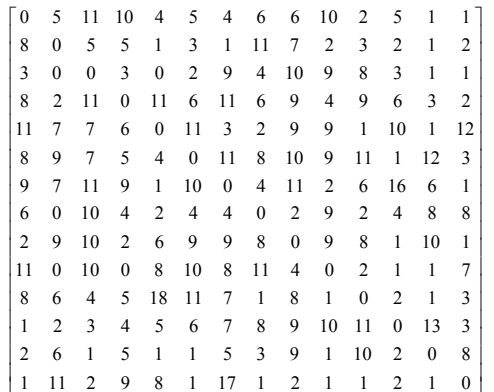

(h)
Fig. 5. Numerical examples: topologies and traffic matrices.

TABLE I COMPARE LTA WITH ILP

\begin{tabular}{c|c|c|c|c|c|c}
\hline \hline Topology & (a) $N=10$ & (b) $N=10$ & (c) $N=10$ & (d) $N=10$ & (e) $N=10$ & (f) $N=14$ \\
\hline ILP & 13 & 14 & 15 & 14 & 14 & 27 \\
\hline LTA & 14 & 15 & 16 & 15 & 16 & 30 \\
\hline Best $r$ & $2,5,7$ & 3,7 & 5 & 6 & 3 & 9,13 \\
\hline Gap & $7.69 \%$ & $7.14 \%$ & $6.67 \%$ & $7.14 \%$ & $14.29 \%$ & $11.11 \%$ \\
\hline$W_{\text {ILP }}$ & 4 & 3 & 4 & 5 & 4 & 4 \\
\hline$W_{\text {LTA }}$ & 4 & 4 & 5 & 5 & 5 & 5 \\
\hline \hline
\end{tabular}

TABLE II LTA SOLUTION FOR TOPOLOGY (a)

\begin{tabular}{c|c|l|c}
\hline \hline No. & \multicolumn{1}{|c|}{ Light-trail } & \multicolumn{1}{|c}{ Accommodated node pairs } & Capacity \\
\hline 1 & $0 \rightarrow 4 \rightarrow 7 \rightarrow 9 \rightarrow 8$ & $(4,9)(0,9)(4,8)(0,8)(7,8)(0,7)(9,8)(4,7)$ & 48 \\
2 & $8 \rightarrow 9 \rightarrow 7 \rightarrow 4 \rightarrow 0$ & $(9,4)(9,0)(8,4)(8,0)(8,7)(7,0)(7,4)$ & 43 \\
3 & $9 \rightarrow 7 \rightarrow 6 \rightarrow 3 \rightarrow 2$ & $(9,7)(9,2)(7,2)(9,6)(7,3)(7,6)$ & 47 \\
4 & $2 \rightarrow 3 \rightarrow 6 \rightarrow 8 \rightarrow 9$ & $(8,9)(2,9)(3,9)(2,8)(6,9)(3,8)(2,3)$ & 46 \\
5 & $2 \rightarrow 1 \rightarrow 5 \rightarrow 7 \rightarrow 9$ & $(7,9)(1,9)(2,7)(5,9)(1,7)(2,5)(5,7)(1,5)$ & 48 \\
6 & $9 \rightarrow 8 \rightarrow 6 \rightarrow 5 \rightarrow 1$ & $(9,5)(8,1)(8,5)(6,1)(8,6)$ & 44 \\
7 & $4 \rightarrow 0 \rightarrow 5 \rightarrow 6 \rightarrow 3$ & $(4,5)(4,3)(0,3)(4,6)(0,6)(5,3)(0,5)$ & 44 \\
8 & $1 \rightarrow 0 \rightarrow 5 \rightarrow 6 \rightarrow 8$ & $(5,8)(1,8)(1,6)(6,8)(5,6)(1,0)$ & 48 \\
9 & $3 \rightarrow 6 \rightarrow 5 \rightarrow 0 \rightarrow 4$ & $(5,4)(3,4)(3,0)(6,4)(6,0)(3,5)(0,4)$ & 43 \\
10 & $4 \rightarrow 0 \rightarrow 5 \rightarrow 1 \rightarrow 2$ & $(4,0)(4,2)(4,1)(0,2)(5,2)(0,1)$ & 48 \\
11 & $3 \rightarrow 6 \rightarrow 7 \rightarrow 5 \rightarrow 1$ & $(7,5)(3,7)(3,1)(6,7)(6,5)(3,6)(5,1)$ & 46 \\
12 & $8 \rightarrow 6 \rightarrow 3 \rightarrow 2 \rightarrow 1$ & $(8,3)(8,2)(6,2)(6,3)(3,2)$ & 43 \\
13 & $2 \rightarrow 1 \rightarrow 5 \rightarrow 0 \rightarrow 4$ & $(1,4)(2,0)(5,0)$ & 12 \\
14 & $0 \rightarrow 1 \rightarrow 2 \rightarrow 3 \rightarrow 6$ & $(2,6)(1,3)(1,2)$ & 19 \\
\hline
\end{tabular}

returns a sub-optimal solution. Specifically, the LTA solution for topology (a) (with traffic matrix (g)) is detailed in TABLE II. Compared to the ILP solution in [6], the number of lighttrails required by LTA is only one more than ILP.

In order to evaluate the efficiency of the reference node mechanism, we compare LTA with an algorithm LTD without reference node. LTD is obtained by slightly modifying LTA as follows: 1) Step 2a) in Fig. 4 is removed; 2) In Step 2b), the most crucial request $t_{a b}$ is identified as the one with the largest
TABLE III LTA vs LTD BASED ON 100 RANDOM TESTS $\left(L_{\max }=4, C=48\right)$

\begin{tabular}{c|c|c|c|c|c|c|c}
\hline \hline$N$ & 10 & 15 & 20 & 25 & 30 & 35 & 40 \\
\hline LTA $_{\text {best }}$ & 12.85 & 32.90 & 66.25 & 114.94 & 183.79 & 270.73 & 380.57 \\
\hline LTA $_{\text {ave }}$ & 13.31 & 34.34 & 68.97 & 119.41 & 189.66 & 278.94 & 390.50 \\
\hline LTD & 13.82 & 34.72 & 69.12 & 119.30 & 189.15 & 278.06 & 388.91 \\
\hline \hline
\end{tabular}

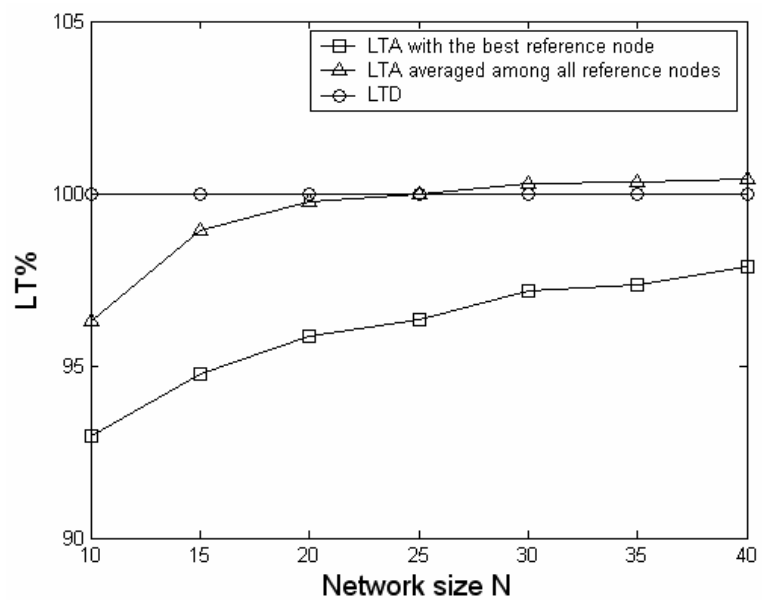

Fig. 6. Reference node efficiency.

$h_{i j}$ and (then) $t_{i j}$, and $d_{i j}{ }^{r}$ is removed; 3) Both Step 2g) and Step 3) are removed and LTD ends at Step 2f), because only one solution is constructed; and 4) $d_{i j}^{r}$ is removed from the packing process in Fig. 3.

TABLE III and Fig. 6 give the results averaged over 100 randomly generated topologies and traffic matrices. The topologies and the traffic matrices are generated as follows.

- Topology construction. We first treat the topology as a directed graph. Each node emanates 2 directed edges to others with the same probability. Then, each directed edge is replaced by a bidirectional edge. We also check the topology to ensure it is connected.

- Traffic matrix generation. $\boldsymbol{T}=\left\{t_{i j}\right\}$ is first generated with $t_{i j}$ uniformly distributed in $[0,12]$. Then we adjust $\boldsymbol{T}$ to characterize some "hot" paths as follows. If $t_{i j}=12$, we reset it to 0 ; if $t_{i j}=11$, we replace it by another random number in $[0,30]$. Then, the traffic matrix preprocessing in Fig. 1 is applied to $\boldsymbol{T}$. For simplicity, we set $t_{i j}=0$ if it is greater than $C=48$ after traffic matrix preprocessing.

In TABLE III, the $2^{\text {nd }}$ and the $3^{\text {rd }}$ rows give the number of light-trails required by LTA using the best reference node and averaged over the $N$ solutions $\left\{\boldsymbol{S o l}_{\boldsymbol{r}} \mid 0 \leq r<N\right\}$, respectively. LTD results are given in the $4^{\text {th }}$ row. TABLE III also translates into Fig. 6, where percentage is used with $100 \%$ for LTD. From Fig. 6, we can see that the gain of LTA over LTD is more significant for small networks. For $N>25$, though $\mathrm{LTA}_{\text {ave }}$ is a little bit larger than LTD, LTA $_{\text {best }}$ is still much smaller. This is because request discreteness in small networks can be efficiently suppressed by reference node. For large-sized networks, the efficiency fades out.

\section{DISCUSSION}

Generally, it is difficult to determine the best reference node $r$. Fig. 7 gives an example with $L_{\max }=3$ and $C=48$. We first assume $r=0$. Note that $d_{i j}{ }^{r}=3$ and $h_{i j}=1$ for all the requests. In Fig. $7 \mathrm{a}$, two light-trails are required to carry $\left\{t_{12}=30, t_{34}=18\right\}$ 


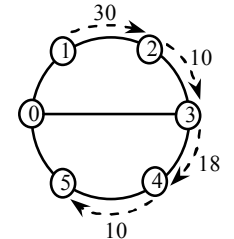

(a)

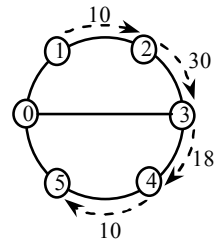

(b)
Fig. 7. The best reference node depends on both topology and traffic matrix.

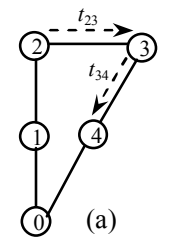

(b)

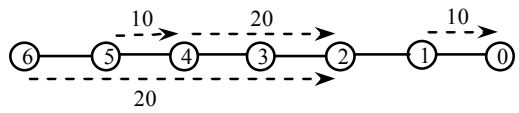

(c)

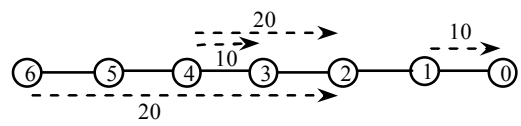

Fig. 8. Intelligence of $d_{i j}{ }^{r}$ definition in (1) $\left(L_{\max }=4, C=48, r=0\right)$.

and $\left\{t_{23}=10, t_{45}=10\right\}$ according to LTA. But, if we exchange $t_{12}$ and $t_{23}$ as in Fig. $7 \mathrm{~b}$, then a light-trail is required to carry $\left\{t_{23}=30, t_{34}=18\right\}$, and $\left\{t_{12}=10\right\}$ and $\left\{t_{45}=10\right\}$ need two additional light-trails due to request discreteness. On the other hand, if $r=5$, only two light-trails are required for both cases in Figs. 7a \& 7b. This example shows that the best reference node depends on both topology and traffic matrix.

Two-dimension coordinates can do a better job in quantifying request discreteness. For example, the discreteness among the requests with the same $d_{i j}{ }^{r}$ is ignored in Fig. 7. Therefore, the reference node mechanism in LTA can only partially address the request discreteness issue. On the other hand, introducing two-dimension coordinates will dramatically complicate the algorithm.

We define $d_{i j}^{r}=h_{i r}+h_{j r}$ as in (1) instead of $d_{i j}{ }^{r}=\max \left\{h_{i r}, h_{j r}\right\}$. With the definition $d_{i j}{ }^{r}=h_{i r}+h_{j r}$, we can identify the most crucial request in a more intelligent way than $d_{i j}{ }^{r}=\max \left\{h_{i r}, h_{j r}\right\}$. This is shown by the three scenarios in Fig. 8, where $L_{\max }=4, C=48$ and $r=0$. In Fig. 8a, $t_{23}$ physically locates farther away from node 0 than $t_{34}$. In this case, $d_{i j}{ }^{r}=h_{i r}+h_{j r}$ can properly return $t_{23}$ as the most crucial request. But with $d_{i j}{ }^{r}=\max \left\{h_{i r}, h_{j r}\right\}$, this will fail because $d^{0}{ }_{23}$ and $d_{34}^{0}$ will take the same value of 2 .

Figs. $8 \mathrm{~b} \& 8 \mathrm{c}$ further show the intelligence of our $d_{i j}{ }^{r}$ definition. The two scenarios differ from each other only by the location of one request: $t_{54}$ in Fig. $8 \mathrm{~b}$ and $t_{43}$ in Fig. 8c. With $d_{i j}{ }^{r}=h_{i r}+h_{j r}, t_{54}$ (instead of $t_{62}$ ) in Fig. $8 \mathrm{~b}$ is identified as the most crucial request, whereas $t_{62}$ is identified in Fig. 8c. Since $t_{54}$ is the most crucial request in Fig. $8 \mathrm{~b}$, it shares a common lighttrail with $t_{62}$. As a result, the remaining not-yet-carried requests $t_{42}$ and $t_{10}$ can share a common light-trail. Similarly, since $t_{62}$ is the most crucial request in Fig. 8c, $\left\{t_{62}, t_{42}\right\}$ and $\left\{t_{43}, t_{10}\right\}$ require two light-trails. In both scenarios, two light-trails are sufficient. However, if $d_{i j}{ }^{r}$ is defined by $d_{i j}{ }^{r}=\max \left\{h_{i r}, h_{j r}\right\}$, then $t_{62}$ will be the most crucial request in both Figs. $8 \mathrm{~b} \& 8 \mathrm{c}$. According to the packing process $\operatorname{pack}(l)$ in Fig. $3, t_{62}$ in Fig. $8 \mathrm{~b}$ will share a light-trail with $t_{42}$ instead of $t_{54}$. As a result, the remaining not-yet-carried requests $t_{54}$ and $t_{10}$ need two additional light-trails (instead of only one). Compared with $d_{i j}{ }^{r}=h_{i r}+h_{j r}$, the definition $d_{i j}{ }^{r}=\max \left\{h_{i r}, h_{j r}\right\}$ leads to a worse solution for Fig. 8b.

The reason behind the above differences is as follows. A request with smaller $h_{i j}$ (such as $t_{54}$ in Fig. $8 \mathrm{~b}$ and $t_{43}$ in Fig. 8c) generally has less opportunity to be carried by the current lighttrail in the packing process $\operatorname{pack}(l)$. If it is far from $r$ (such as $t_{54}$ in Fig. 8b), then it may boost request discreteness. Therefore, we should give it higher priority and accommodate it first. Although $t_{62}$ physically locates farther away from $r=0$ than $t_{54}$, it is advantageous to have a larger $h_{i j}$ and thus being preferred by pack $(l)$.

In LTA, we have constructed all the light-trails with the same length limit $L_{\max }$. We can check through the final solution to see if some light-trails can be shortened. For example, in Fig. $7 \mathrm{~b}$, the light-trail carrying $\left\{t_{23}=30, t_{34}=18\right\}$ can be shortened to 2 hops although $L_{\max }=3$.

\section{CONCLUSION}

In this paper, we analyzed the key factors that affect the solution of the light-trail assignment problem. These factors include the request discreteness, the shortest path length of each request, and the flow volume. Taking all the three factors into account, we proposed an intelligent heuristic algorithm LTA (Light-Trail Assignment) to minimize the number of light-trails required. Notably, LTA adopts a reference node mechanism to address the request discreteness issue. To the best of our knowledge, LTA is the first heuristic proposed in this area. It was shown to be very efficient by always returning a sub-optimal solution.

\section{REFERENCES}

[1] M. Mahony, D. Simeonidou, D. Hunter and A. Tzanakaki, "The application of optical packet switching in future communication networks", IEEE Communications Magazine, pp. 128-135, Mar. 2001.

[2] C. Qiao and M. Yoo, "Optical burst switching (OBS) - a new paradigm for an optical internet", J. High Speed Networks (JHSN), vol. 8, issue 1, pp. 69-84, 1999.

[3] A. Gumaste and I. Chlamtac, "Light-trails: a novel conceptual framework for conducting optical communications", IEEE HPSR '03, pp. 251-256, Jun. 2003.

[4] I. Chlamtac and A. Gumaste, "Light-trails: A solution to IP centric communication in the optical domain", Online publication: February 17, 2003, Springer-Verlag Berlin Heidelberg 2003.

[5] M. T. Frederick, N. A. VanderHorn, and A. K. Somani, "Light trails: a sub-wavelength solution for optical networking”, IEEE HPSR '04, pp. 175-179, Apr. 2004.

[6] Jing Fang, Wensheng He, and A. K. Somani, "Optimal light trail design in WDM optical networks", IEEE ICC '04, vol. 3, pp. 1699-1703, Jun. 2004.

[7] A. Gumaste, G. Kuper and I. Chlamtac, "Optimizing light-trail assignment to WDM networks for dynamic IP centric traffic", IEEE LANMAN'04, pp. 113-118, Apr. 2004.

[8] A. Gumaste, I. Chlamtac, and J. P. Jue, "Light-frames: a pragmatic framework for optical packet transport', IEEE ICC '04, vol. 3, pp. 15371542, Jun. 2004.

[9] Yabin Ye, H. Woesner, R. Grasso, Tao Chen and I. Chlamtac, "Traffic grooming in light trail networks", IEEE GLOBECOM '05, vol. 4, pp. 1957-1962, Dec. 2005.

[10] Weiyi Zhang, Guoliang Xue, Jian Tang and K. Thulasiraman, "Dynamic light trail routing and protection issues in WDM optical networks", IEEE GLOBECOM'05, vol. 4, pp. 1963-1967, Dec. 2005.

[11] D. Kliazovich, F. Granelli, H. Woesner and I. Chlamtac, "Bidirectional light-trails for synchronous communications in WDM networks", IEEE GLOBECOM'05, vol. 4, pp. 1947-1951, Dec. 2005.

[12] R. K. Ahuja, T. L. Magnanti, and J. B. Orlin, Network Flows: Theory, Algorithms, and Applications. Upper Saddle River, NJ: Prentice-Hall, 1993. 